\title{
Revealing evolved massive stars with Spitzer, WISE and SALT
}

\author{
A. Kniazev ${ }^{* \dagger}$ \\ South African Astronomical Observatory, PO Box 9, 7935, South Africa \\ Southern African Large Telescope Foundation, PO Box 9, 7935, PO Box 9, 7935, South Africa \\ E-mail: akniazevesaao.ac.za

\section{Gvaramadze} \\ Sternberg Astronomical Institute, Lomonosov Moscow State University, Moscow 119992, Russia \\ Space Research Institute, Russian Academy of Sciences, Profsoyuznaya 84/32, 117997 Moscow, \\ Russia \\ E-mail: vgvaram@mx.iki.rssi.ru
}

\begin{abstract}
We present the results of optical spectroscopic observations of 55 candidate evolved massive stars revealed through the detection of mid-infrared nebulae of various shapes surrounding them with the Spitzer Space Telescope and Wide-field Infrared Survey Explorer. These observations, carried out with the Southern African Large Telescope (SALT) in 2010-2015, led to the discovery of about two dozens emission-line stars, of which 15 stars we classify as candidate luminous blue variables (cLBVs). Spectroscopic and photometric monitoring revealed significant changes in the spectra and brightness of four newly identified cLBVs, meaning that they are new members of the class of bona fide LBVs. We present an updated list of the Galactic bona fide LBVs. Currently, this list contains eighteen stars, of which more than 70 per cent are associated with circumstellar nebulae. We also discovered a very rare [WN] star - the central star of the planetary nebula Abell 48, and a WN3 star in a close, eccentric binary system with an O6 V star in the Large Magellanic Cloud - the first-ever extragalactic massive star identified via detection of a circular shell around it. Most of the remaining targets are tentatively classified as OB, A and M stars.
\end{abstract}

SALT Science Conference 2015 -SSC2015-

1-5 June, 2015

Stellenbosch Institute of Advanced Study, South Africa

\footnotetext{
* Speaker.

${ }^{\dagger}$ This paper uses observations made with the Southern African Large Telescope (SALT).
} 


\section{Introduction}

Stars more massive than $\geq 20 M_{\odot}$ experience the short-lived luminous blue variable (LBV) stage [4], which among other evolutionary stages of massive stars is the most interesting in observational manifestations and perhaps the most important in the evolutionary sense [20, 25, 34, 8]. During this stage a massive star exhibits irregular spectroscopic and photometric variability on time-scales from years to decades or longer, which is reflected in changes of the stellar type from late O/early B supergiants to A/F-type ones (see e.g. [29, 7]) and changes in the brightness by several magnitudes. At the brightness maximum, LBVs could be confused with supernovae (e.g. $[6,35]$ ), and it is believed that some LBVs could be the direct progenitors of supernovae (e.g. $[24,8])$. The LBV stars experience episodes of enhanced, sometimes eruptive, mass loss, so that most of them (see $[3,22]$ and Table 2$)$ are surrounded by compact $(\sim 0.1-1 \mathrm{pc}$ in diameter) shells with a wide range of morphologies (e.g. [27, 39, 12]).

The LBV phenomenon is still ill-understood, which is mostly because the LBV stars are very rare objects. The recent census of Galactic confirmed and candidate LBVs (cLBVs) presented in [36] lists only 13 and 25 stars, respectively. The discovery of additional LBVs would, therefore, be of great importance for understanding their evolutionary status and their connection to other massive transient stars, as well as for unveiling the driving mechanism(s) of the LBV phenomenon.

Detection of LBV-like shells can be considered as an indication that their associated stars are massive and evolved, and therefore could be used for the selection of candidate massive stars for follow-up spectroscopy. Because of the huge interstellar extinction in the Galactic plane, the most effective channel for the detection of circumstellar shells is through imaging with modern infrared (IR) telescopes. Application of this approach using the Spitzer Space Telescope and Wide-field Infrared Survey Explorer (WISE) resulted in the discovery of hundreds of such shells whose central stars could be LBVs or other types of evolved massive stars ([12, 37, 26, 13]). Indeed, follow-up optical and IR spectroscopy of these central stars led to the discovery of dozens of new cLBV, blue supergiant and Wolf-Rayet (WR) stars in the Milky Way ([9, 10, 11, 12, 37, 38, 14, 30, 31, 2, 5, $15,16,17,22,18,19,23])$. Because of reddening many of the central stars are very dim in the optical, which makes inevitable the use of 8-10-m class telescopes like the Southern African Large Telescope (SALT). Here we report the results of optical spectroscopy with the SALT of 55 central stars of compact mid-IR nebulae discovered with Spitzer and WISE.

\section{Observations}

The SALT observations were carried out in 2010-2015 with the Robert Stobie Spectrograph (RSS; [1]) in the long-slit mode. In most cases, the spectra covered the range of 4200-7300. The primary reduction of the data was done with SALT science pipeline. After that, the bias and gain corrected and mosaicked long-slit data were reduced in the way described in [21]. Examples of 1D finally reduced spectra of a dozen emission-line stars are shown in Figure 1, while Figure 2 presents the mid-IR images of circumstellar nebulae associated with these stars. The list of all observed targets is given in Table 1 .

To search for possible spectroscopic and photometric variability of the newly identified cLBVs, we obtained additional spectra with the SALT and performed photometric monitoring of these stars 


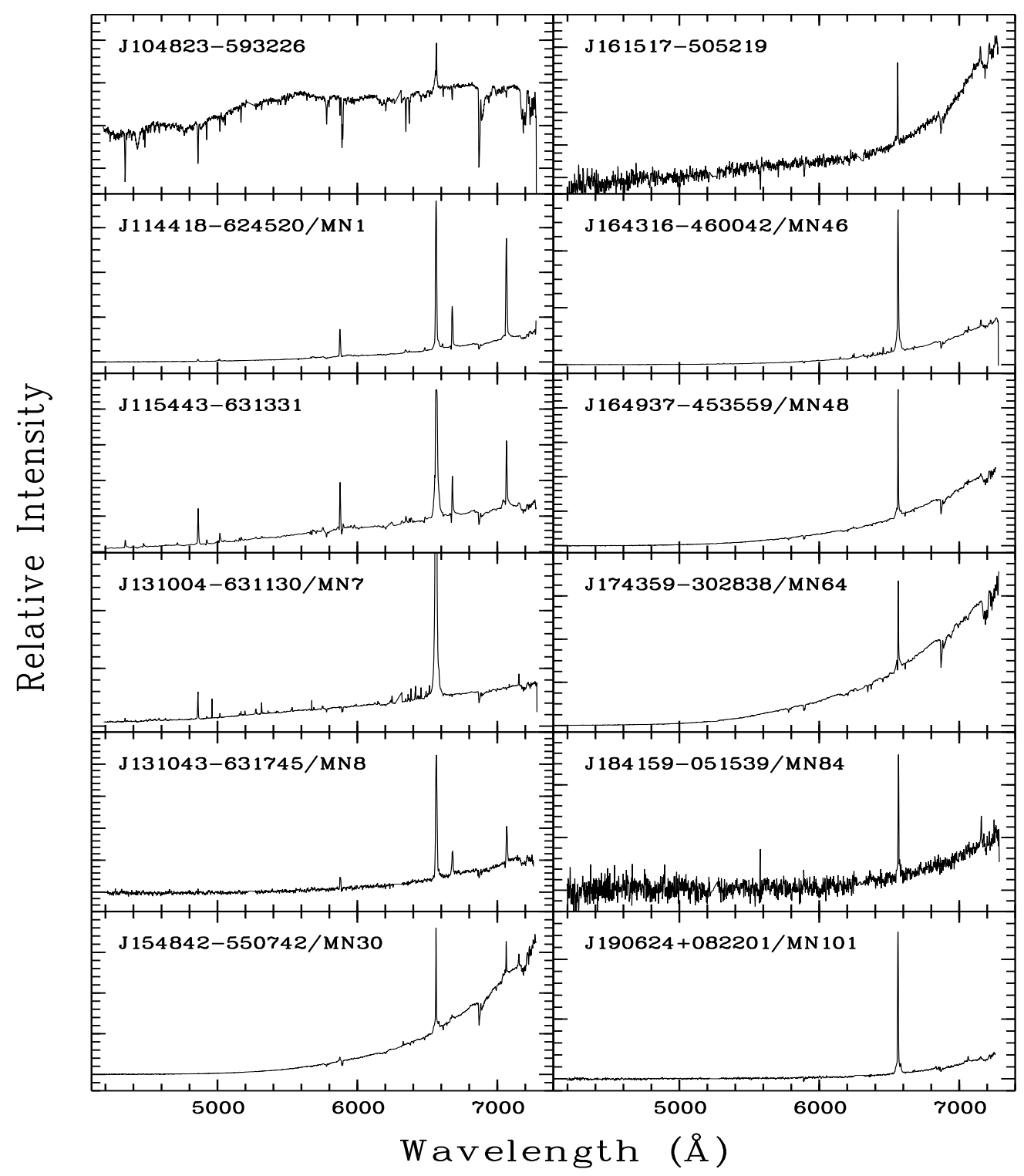

Figure 1: The observed SALT spectra of a dozen emission-line stars from our sample of candidate evolved massive stars revealed with Spitzer and WISE.

with the 76-cm telescope of the South African Astronomical Observatory.

\section{Results}

We carried out optical SALT spectroscopy of 55 candidate evolved massive stars. The first results of our observing program were presented in $[14,32,16,17,18,22,19]$. Table 1 summarizes the (mostly preliminary) spectral classification of the observed targets. We detected about two dozen of emission-line stars, of which 15 stars were classified as cLBVs. Subsequent spectroscopic 


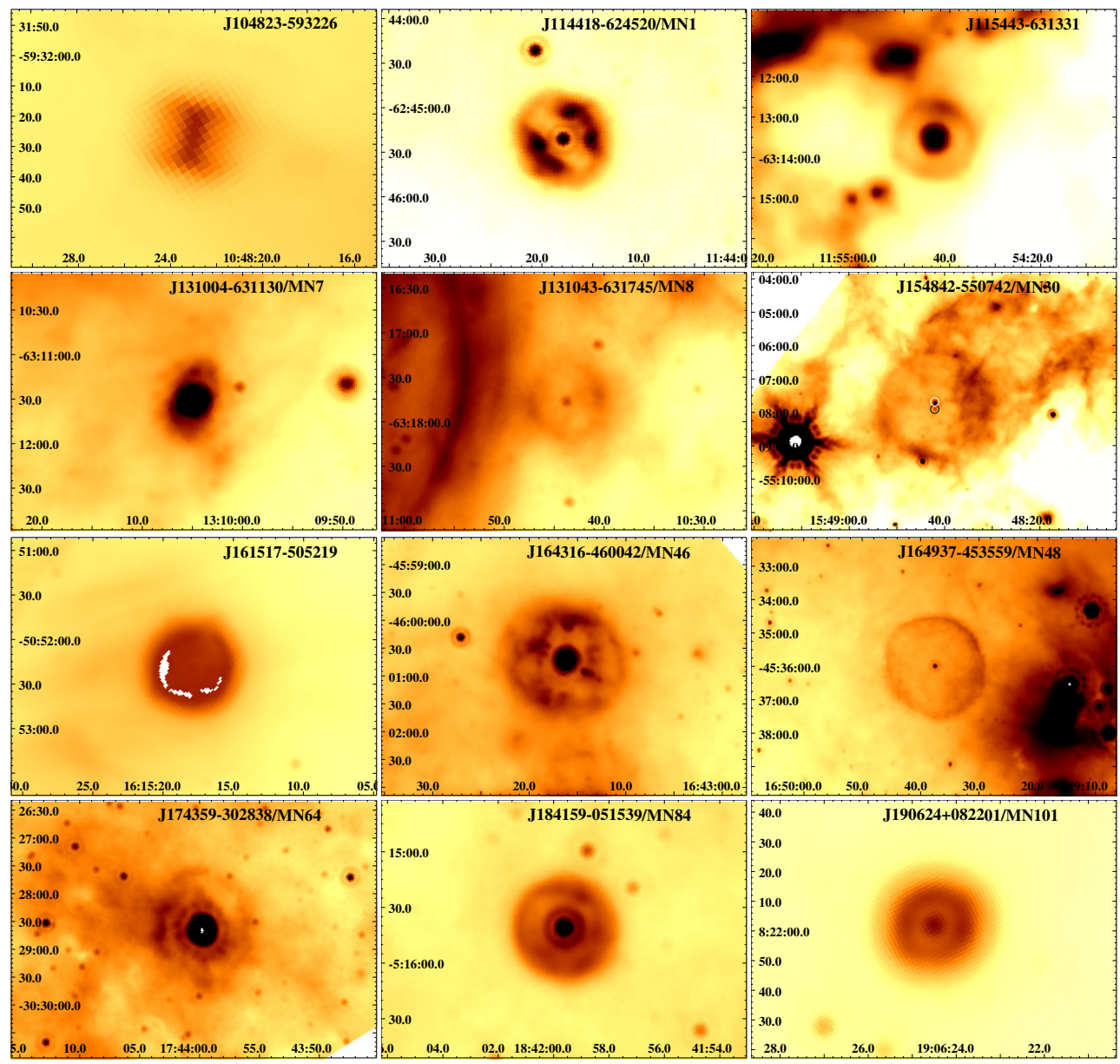

Figure 2: Mid-IR images of circumstellar nebulae around stars whose spectra are shown in Figure 1. All but one of these nebulae were discovered with Spitzer at $24 \mu \mathrm{m}$. The nebula around J115443-631331 was discovered with WISE at $22 \mu \mathrm{m}$. The nebula shown in the right panel of the second row contains two stars near the geometrical centre. One of them (marked by a white circle) is the cLBV J154842-550742/MN30. The second one (marked by a black circle) is the WC9 star J154842-550755. The coordinates are in units of RA(J2000) and Dec.(J2000) on the horizontal and vertical scales, respectively.

and photometric monitoring of these stars allowed us to confirm the LBV status of four of them (see Tables 1 and [17, 22, 19, 23]). Figure 3 shows the evolution of the spectrum of Wray 16-137 (one of the four newly identified Galactic bona fide LBVs) in 2011-2014. One can see that the He I emission lines have almost disappeared, while numerous Fe II emissions have become prominent. These changes along with significant brightness increase of the star (by about 1 mag during three years) indicate that currently Wray 16-137 experiences an S Dor-like outburst.

We also discovered a rare WN-type central star of a planetary nebula (Abell 48), which is the second known example of [WN] stars [32]. Thanks to the high angular resolution of Spitzer images (6 $\operatorname{arcsec}$ at $24 \mu \mathrm{m}$ ), we detected a new circular shell in the Large Magellanic Cloud. Follow up spectroscopy of its central star with SALT (and several other telescopes) resulted in the discovery of a new WR star in a close, eccentric binary system with an O6 V star [16]. The majority of the 
Table 1: The observed central stars of mid-IR nebulae and their (mostly preliminary) spectral classification. The stars whose spectra and nebulae are presented in Figures 1 and 2 are starred.

\begin{tabular}{|c|c|c|c|c|c|}
\hline $\begin{array}{l}\text { Object } \\
\text { (1) }\end{array}$ & $\begin{array}{c}\text { Name } \\
(2)\end{array}$ & $\begin{array}{l}\text { Type } \\
\text { (3) }\end{array}$ & $\begin{array}{l}\text { Object } \\
\text { (1) }\end{array}$ & $\begin{array}{l}\text { Name } \\
(2)\end{array}$ & $\begin{array}{l}\text { Type } \\
\text { (3) }\end{array}$ \\
\hline J045304-692352 & BAT993a & $\mathrm{WN} 3 \mathrm{~b}+\mathrm{O} 6 \mathrm{~V}^{a}$ & J153856-563722 & MN25 & OB \\
\hline J052848-705105 & & OB & J154527-535602 & MN26 & OB \\
\hline J052412-683011 & LHA $120 \mathrm{~N}$ & OB & J154842-550742* & MN30 & $\mathrm{cLBV}^{i}$ \\
\hline J071810-265124 & CD-264148 & OB & J154842-550755 & & $\mathrm{WC}^{j}$ \\
\hline J091714-495502 & & M & J161132-512906 & MN40 & OB \\
\hline J100122-550046 & & OB & $\mathrm{J} 161517-505219^{*}$ & & $\mathrm{cLBV}^{k}$ \\
\hline J103638-580012 & & $\mathrm{Be}$ & J163239-494213 & MN44 & $\mathrm{LBV}^{l}$ \\
\hline $\mathrm{J} 104823-593226^{*}$ & HD 93795 & $\mathrm{~A}$ & J164316-460042* & MN46 & $\mathrm{cLBV}^{m}$ \\
\hline J110340-592559 & HD 96042 & OB & J164937-453559* & MN48 & $\mathrm{LBV}^{n}$ \\
\hline J111428-611820 & Wray $15-780$ & $\mathrm{OB}$ & J170723-395651 & MN50 & $\mathrm{M}^{o}$ \\
\hline $\mathrm{J} 114418-624520^{*}$ & MN1 & $\mathrm{cLBV}^{b}$ & J171307-384734 & CD-38 11646 & $\mathrm{OB}$ \\
\hline J115443-631331* & & cLBV & J172031-330949 & WS2 & $\mathrm{cLBV}^{p}$ \\
\hline J120058-631259 & MN2 & OB & J173753-302311 & & OB \\
\hline J124626-632427 & WRAY 17-56 & $\mathrm{PN}^{c}$ & J173918-312424 & MN59 & $\mathrm{OB}^{q}$ \\
\hline J131004-631130* & MN7 & cLBV & J174359-302838* & MN64 & $\mathrm{cLBV}^{r}$ \\
\hline J131028-621331 & & OB & J174627-302001 & & OB \\
\hline $\mathrm{J} 131043-631745^{*}$ & MN8 & $\mathrm{cLBV}^{d}$ & J180433-210326 & HD 313642 & A \\
\hline J131933-623844 & MN10 & $\mathrm{OB}^{e}$ & J180612-211745 & MN74 & OB \\
\hline J132647-615924 & & M & J180823-221939 & ALS 4684 & OB \\
\hline J133628-634538 & WS1 & $\operatorname{LBV}^{f}$ & J182721-132209 & & OB \\
\hline J133654-632552 & & OB & J183217-091614 & & $\mathrm{OB}^{s}$ \\
\hline J135015-614855 & Wray 16-137 & $\mathrm{LBV}^{g}$ & J183528-064415 & & OB \\
\hline J140707-652934 & CPD-64 2731 & $\mathrm{O}$ & J184159-051539* & MN84 & $\mathrm{cLBV}^{t}$ \\
\hline J143111-610202 & MN14 & OB & J184246-031317 & & [WN5] $]^{u}$ \\
\hline J145409-564817 & TYC 8689-1568-1 & $\mathrm{Be}$ & $\mathrm{J} 185404+033544$ & & $\mathrm{M}$ \\
\hline J151342-585318 & MN17 & OB & J190421+060001 & MN100 & $\mathrm{OB}^{v}$ \\
\hline J151641-582226 & MN18 & $\mathrm{B} 1 \mathrm{Ia}^{h}$ & J190624+082201* & MN101 & $\mathrm{cLBV}^{w}$ \\
\hline J151959-572415 & MN19 & $\mathrm{OB}$ & & & \\
\hline \multicolumn{6}{|c|}{$\begin{array}{l}\text { Notes: }{ }^{a}[16] ;{ }^{b} \text { classified as an Oe/WN in [37]; }{ }^{c} \text { originally classified as a PN in [28]; }{ }^{d} \text { classified as } \\
\text { an Oe/WN in [37]; }{ }^{e} \text { originally classified as an OB in [38]; }{ }^{f}[14,22] ;{ }^{g}[17] ;{ }^{h}[18] ;{ }^{i} \text { classified as a } \\
\text { Be/B[e]/LBV in [38]; }{ }^{j} \text { originally classified as a WC9 in [38]; }{ }^{k} \text { classified as a star in transition from AGB } \\
\text { to PN in [33]; }{ }^{l} \text { [19]; }{ }^{m} \text { [12]; }{ }^{n} \text { [23]; }{ }^{o} \text { classified as a M1 I in [37]; }{ }^{p} \text { [14]; }{ }^{q} \text { originally classified as an OB } \\
\text { in [37]; }{ }^{r} \text { classified as a Be in [37] and as an OB in [38]; }{ }^{s} \text { classified as a BA in [38]; }{ }^{t} \text { classified as a } \\
\text { Be/B[e]/LBV in [37] and as a cLBV in [31]; }{ }^{u} \text { originally classified as a WN6 in [37] and then re-classified } \\
\text { as a [WN5] in [32]; }{ }^{v} \text { classified as a FG in [38]; }{ }^{w} \text { classified as B Be]/LBV in [38] and as a cLBV in [31]. }\end{array}$} \\
\hline
\end{tabular}




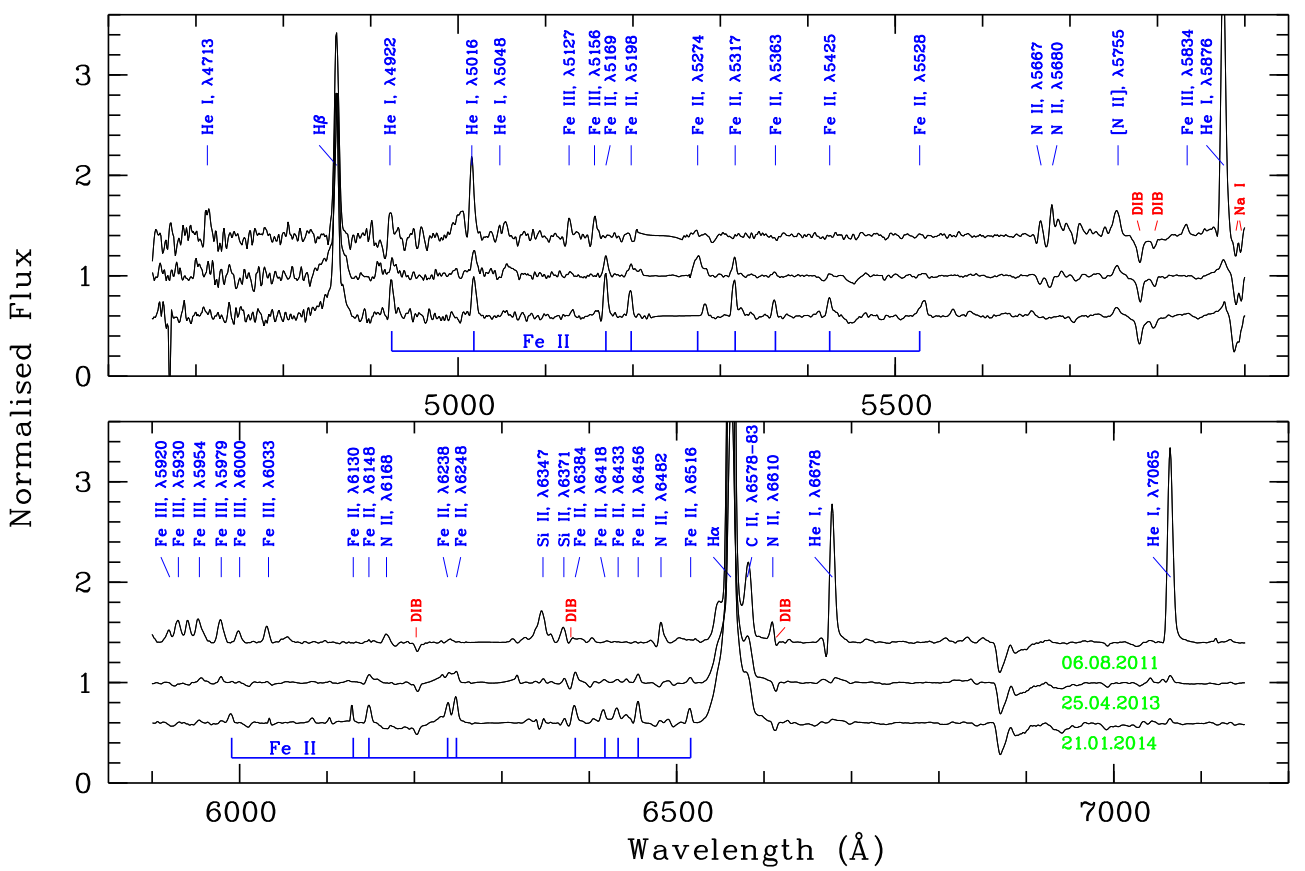

Figure 3: Evolution of the (normalized) spectrum of the new Galactic bona fide LBV Wray 16-137 in 2011-2014 (adopted from [17]).

Table 2: Current census of the Galactic bona fide LBVs. The objects with detected circumstellar nebulae are starred.

\begin{tabular}{llll}
\hline HR Car* & $\eta$ Car* & AGCar* & Wray 15-751* \\
[GKM2012] WS1* & Wray 16-137* & [GKF2010] MN44* & Cl* Westerlund 1W 243 \\
[GKF2010] MN48* & HD 160529 & GCIRS 34W & {$[$ MMC2010] LBV G0.120-0.048* } \\
qF362 & HD 168607 & MWC 930* & G24.73+0.69* \\
AFGL 2298* & PCyg* & & \\
\hline
\end{tabular}

remaining targets were tentatively classified as $\mathrm{OB}, \mathrm{A}$ and $\mathrm{M}$ stars.

Finally, we present in Table 2 the current census of the Galactic bona fide LBVs (eighteen stars in total). The objects with detected circumstellar nebulae are starred. As follows from the table, 72 per cent of the Galactic confirmed LBVs are associated with nebulae. This provides further proof that the detection of compact mid-IR shells is a powerful tool for identifying (candidate) LBVs. Searches for new mid-IR nebulae continue, with further discoveries of LBVs and other related stars anticipated.

\section{Acknowledgments}

The observations reported in this paper were obtained with the SALT programs 2010-1-RSA_OTH-001, 2011-3-RSA_OTH-002, 2013-1-RSA_OTH-014, 2013-2-RSA_OTH-003 and 2015-1-SCI-017. AYK 
acknowledges support from the National Research Foundation (NRF) of South Africa. VVG acknowledges the Russian Science Foundation grant 14-12-01096. This work was partially supported by the Russian Foundation for Basic Research grant 16-02-00148. We are grateful to referees for useful suggestions on the manuscript.

\section{References}

[1] E.B. Burgh et al., Prime focus imaging spectrograph for the Southern African Large Telescope: optical design, SPIE 4841 (2003) 1463

[2] S. Burgmeister et al. WR 120bb and WR 120bc: a pair of WN9h stars with possibly interacting circumstellar shells, MNRAS $\mathbf{4 2 9}$ (2013) 3305

[3] J.S. Clark, V.M. Larionov, A. Arkharov, On the population of galactic Luminous Blue Variables, A\&A 435 (2005) 239

[4] P.S. Conti, Basic observational constraints on the evolution of massive stars, in proceedings of Observational Tests of the Stellar Evolution Theory (1984) 233

[5] N. Flagey et al., Palomar/TripleSpec observations of Spitzer/MIPSGAL $24 \mu \mathrm{m}$ circumstellar shells: unveiling the natures of their central sources, AJ 148 (2014) 34

[6] R. W. Goodrich et al., SN 1961V - an extragalactic Eta Carinae analog, ApJ 342 (1989) 908

[7] J.H. Groh et al., Bona fide, strong-variable Galactic luminous blue variable stars are fast rotators: detection of a high rotational velocity in HR Carinae, ApJ 705 (2009) L25

[8] J.H. Groh et al., Massive star evolution: luminous blue variables as unexpected supernova progenitors, A\&A $\mathbf{5 5 0}$ (2013) L7

[9] V.V. Gvaramadze et al., Discovery of a new Wolf-Rayet star and its ring nebula in Cygnus, MNRAS 400 (2009) 524

[10] V.V. Gvaramadze et al., A new Wolf-Rayet star and its circumstellar nebula in Aquila, MNRAS $\mathbf{4 0 3}$ (2010a) 760

[11] V.V. Gvaramadze et al., MN112: a new Galactic candidate luminous blue variable, MNRAS 405 (2010b) 520

[12] V.V. Gvaramadze, A.Y. Kniazev, S. Fabrika, Revealing evolved massive stars with Spitzer, MNRAS 405 (2010c) 1047

[13] V.V. Gvaramadze et al., Search for OB stars running away from young star clusters. II. The NGC 6357 star-forming region, $A \& A \mathbf{5 3 5}$ (2011) A29

[14] V.V. Gvaramadze et al., Discovery of two new Galactic candidate luminous blue variables with Wide-field Infrared Survey Explorer, MNRAS 421 (2012) 3325

[15] V.V. Gvaramadze et al., TYC 3159-6-1: a runaway blue supergiant, MNRAS 437 (2014) 2761

[16] V.V. Gvaramadze et al., Discovery of a new Wolf-Rayet star and a candidate star cluster in the Large Magellanic Cloud with Spitzer, MNRAS 442 (2014) 929

[17] V.V. Gvaramadze et al., Discovery of a new Galactic bona fide luminous blue variable with Spitzer, MNRAS 445 (2014) L84

[18] V.V. Gvaramadze et al., The blue supergiant MN18 and its bipolar circumstellar nebula, MNRAS 454 (2015) 219 
[19] V.V. Gvaramadze, A.Y. Kniazev, L. Berdnikov, Discovery of a new bona fide luminous blue variable in Norma, MNRAS 454 (2015) 3710

[20] R.M. Humphreys, K. Davidson, The luminous blue variables: astrophysical geysers, PASP 106 (1994) 1025

[21] A.Y. Kniazev et al., The metallicity extremes of the Sagittarius dSph: SALT spectroscopy of PNe, MNRAS 388 (2008) 1667

[22] A.Y. Kniazev, V.V. Gvaramadze, L.N. Berdnikov, WS1: one more new Galactic bona fide luminous blue variable, MNRAS 449 (2015) L60

[23] A.Y. Kniazev, V.V. Gvaramadze, L.N. Berdnikov, in preparation (2016)

[24] R. Kotak, J.S. Vink, Luminous blue variables as the progenitors of supernovae with quasi-periodic radio modulations, $A \& A \mathbf{4 6 0}$ (2006) L5

[25] N. Langer et al., Towards an understanding of very massive stars. A new evolutionary scenario relating O stars, LBVs and Wolf-Rayet stars, A\&A 290 (1994) 819

[26] D.R. Mizuno et al., A catalog of MIPSGAL disk and ring sources, AJ 139 (2010) 1542

[27] A. Nota et al., Nebulae around luminous blue variables: a unified picture, ApJ 448 (1995) 788

[28] Q.A. Parker et al., The Macquarie/AAO/Strasbourg H $\alpha$ planetary nebula catalogue: MASH, MNRAS 373 (2006) 79

[29] O. Stahl et al., Long-term spectroscopic monitoring of the luminous blue variable AG Carinae, A\&A 375 (2001) 54

[30] G.S. Stringfellow et al., New Galactic candidate luminous blue variables and Wolf-Rayet stars, in proceedings of From Interacting Binaries to Exoplanets: Essential Modeling Tools, IAUS 282 (2012) 267

[31] G.S. Stringfellow et al., Spectral identification of new Galactic cLBV and WR Stars, in proceedings of Scientific Meeting in Honor of Anthony F. J. Moffat held at Auberge du Lac Taureau, ASPC 465(2012) 514

[32] H. Todt et al., Abell 48 - a rare WN-type central star of a planetary nebula, MNRAS 430 (2013) 2302

[33] W.E.C.J. van der Veen, H.J. Habing, T.R. Geballe, Objects in transition from the AGB to the planetary nebula stage - New visual and infrared observations, A\&A 226 (1989) 108

[34] A.M. van Genderen, S Doradus variables in the Galaxy and the Magellanic Clouds, A\&A 366 (2001) 508

[35] S.D. Van Dyk et al., Possible recovery of SN $1961 \mathrm{~V}$ in Hubble Space Telescope archival images, PASP 114 (2002) 700

[36] J.S. Vink, Eta Carinae and the luminous blue variables in proccedings of Eta Carinae and the Supernova Impostors, Astrophys. \& Sp. Sci. Library 384 (2012) 221

[37] S. Wachter et al., A hidden population of massive stars with circumstellar shells discovered with the Spitzer Space Telescope, AJ 139 (2010) 2330

[38] S. Wachter et al. Massive stars with circumstellar shells discovered with the Spitzer Space Telescope, BSRSL 80 (2011) 322

[39] K. Weis, LBV nebulae: The mass lost from the most massive stars, RvMA 14 (2001) 261 